\title{
Structure of the redox sensor domain of Methylococcus capsulatus (Bath) MmoS¥
}

\author{
Uchechi E. Ukaegbu and Amy C. Rosenzweig * \\ Departments of Biochemistry, Molecular Biology and Cell Biology and of Chemistry, Northwestern \\ University, Evanston, IL 60208
}

\begin{abstract}
MmoS from Methylococcus capsulatus (Bath) is the multidomain sensor protein of a two component signaling system proposed to play a role in the copper-mediated regulation of soluble methane monooxygenase (sMMO). MmoS binds an FAD cofactor within its N-terminal tandem Per-ArntSim (PAS) domains, suggesting that it functions as a redox sensor. The crystal structure of the MmoS tandem PAS domains, designated PAS-A and PAS-B, has been determined to 2.34 Åresolution. Both domains adopt the typical PAS domain $\alpha / \beta$ topology and are structurally similar. The two domains are linked by a long $\alpha$ helix and do not interact with one another. The FAD cofactor is housed solely within PAS-A and is stabilized by an extended hydrogen bonding network. The overall fold of PAS-A is similar to other flavin-containing PAS domains, but homodimeric interactions in other structures are not observed in the MmoS sensor, which crystallized as a monomer. The structure both provides new insight into the architecture of tandem PAS domains and suggests specific residues that may play a role in MmoS FAD redox chemistry and subsequent signal transduction.
\end{abstract}

Per-Arnt-Sim (PAS) domains are signaling domains that respond to changes in redox potential, light, concentration of small ligands, and internal energy of a cell $(1,2)$. Widely distributed in proteins across all kingdoms of life, PAS domains often detect signals via an associated cofactor such as heme, flavin, or 4-hydroxycinnamic acid. PAS domains that do not bind cofactors respond to environmental cues by protein-protein interactions with a second PAS domain or an effector protein $(2,3)$. Some PAS domains include C-terminal regions that are similar to the PAS domain and are called PAC motifs (4). Examples of PAS-containing proteins in eukaryotes include circadian clock proteins, voltage-activated ion channels, and hypoxiainducible factor (HIF). In bacteria, PAS domains are typically present in the sensor protein of two component signaling systems (2). In these systems, the PAS domain within the sensor protein detects environmental stimuli, which may result in autophosphorylation of a conserved histidine residue within a histidine kinase domain. The phosphoryl group is then transferred to an aspartic acid residue in the second component, the response regulator protein, mediating changes in gene expression and cell behavior (5-7).

MmoS from the methanotroph Methylococcus capsulatus (Bath) is a PAS domaincontaining sensor protein that may function in copper-mediated regulation of soluble methane monooxygenase (sMMO) (8-10). Methane monooxygenases (MMOs) catalyze the oxidation of methane to methanol and are potentially useful for bioremediation (11). Two forms of MMO

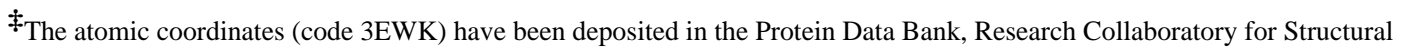
Bioinformatics, Rutgers University, New Brunswick, NJ (http://www.rcsb.org/).

*To whom correspondence may be addressed. Tel.: 847-467-5301; Fax: 847-467-6489; E-mail: E-mail: amyr@ northwestern.edu. SUPPORTING INFORMATION AVAILABLE Gel filtration and analytical ultracentrifugation data are available free of charge via the Internet at http://pubs.acs.org.
} 
exist in nature. All methanotrophs except Methylocella silvestris BL2 $(12,13)$ express a particulate, membrane-bound form (pMMO) that contains copper $(9,14)$. A small subset of methanotrophs, including M. capsulatus (Bath), produce an iron-containing, soluble form (sMMO) $(15,16)$ at low copper-to-biomass ratios $(17,18)$.

The mechanism by which copper starvation leads to sMMO expression in these methanotrophs is not understood, but is hypothesized to involve the proteins encoded by four genes downstream of the sMMO operon, $m m o R, m m o G, m m o Q$, and $m m o S(8)$. MmoR is a transcription factor that likely activates sMMO expression at low copper levels. MmoG is similar to GroEL and is proposed to facilitate the proper folding and assembly of MmoR and/ or the sMMO complex. Marker-exchange mutagenesis of these two proteins in $M$. capsulatus (Bath) and a second organism, Methylosinus trichosporium OB3b, indicates that sMMO transcription is dependent on the presence of both MmoG and MmoR $(8,19)$. MmoS and $\mathrm{MmoQ}$ correspond to the sensor and regulator components of a two-component system. Since the MmoR sequence has no obvious copper-binding motifs, MmoS and MmoQ have been suggested to sense copper levels and relay the signal to MmoR (8).

The N-terminal region of MmoS comprises a predicted $\mathrm{N}$-terminal transmembrane helix followed by two PAS-PAC domains proposed to function in sensing copper levels $(8,10)$ and a GAF domain (20). The copper signal may modulate the autophosphorylation of a histidine residue in the HisKA domain by the HATPase domain. Two receiver domains (REC) and a His-containing phosphotransfer domain (HPT) at the C-terminus may be involved in transfer of the phosphoryl group to $\mathrm{MmoQ}$ (Figure 1). Biochemical characterization of MmoS revealed the presence of an FAD cofactor with a redox potential of $E_{o}=-290 \pm 2 \mathrm{mV}$ at pH 8.0 and 25 ${ }^{\circ} \mathrm{C}$ (10). Other FAD-containing sensor domains include NifL (21) and Aer (22). On the basis of its similarity to these proteins and the observation that it does not bind copper ions, we proposed that $\mathrm{MmoS}$ might mediate the copper switch via a redox signal (10). One possible scenario is that $\mathrm{MmoS}$ exists in the reduced $\mathrm{FADH}_{2}$ state at low copper levels. In this model, reduced MmoS starts a phosphorylation relay that results in MmoR activation of sMMO transcription as proposed by Murrell and coworkers (8). At high copper concentrations, oxidation of $\mathrm{FADH}_{2}$ to FAD is hypothesized to cause a conformational change in $\mathrm{MmoS}$ and an interaction with MmoR which then inhibits the ability of MmoR to activate transcription of the sMMO genes (10).

Toward understanding the molecular mechanism by which MmoS senses changes in cellular redox levels, we determined the $2.34 \AA$ resolution crystal structure of the MmoS redox sensor domain, consisting of the tandem PAS domains (PAS-A and PAS-B) (Figure 1). Although PAS-containing proteins typically contain multiple PAS domains (2), almost all the available crystal structures represent single PAS domains. The current structure thus provides valuable new insight into the architecture of tandem PAS domains. In addition, the structure reveals key residues that may play a role in FAD redox chemistry and subsequent signal transduction.

\section{MATERIALS AND METHODS}

\section{Expression and Purification of the Sensor Domain}

The gene encoding the M. capsulatus (Bath) MmoS sensor domain, consisting of the two PAS domains spanning residues 85-329, was amplified with PfU polymerase (Invitrogen) from the MmoS $\mathrm{Tm}^{-}$construct described previously (10) using the following primers: forward, 5' GACGACGACAAGATGCAGCGCAACAAGGAGCTCCTGGAC - 3', and reverse, 5' GAGGAGAAGCCCGGTTCACCGGGCGCTCACGTCGTGCTGG - 3'. The gene was transformed into BL21(DE3)pLysS E. coli cells in a pET46 (Novagen) vector that includes an Nterminal (His) 6 tag and an adjacent enterokinase cleavage site. Cultures $(50 \mathrm{~mL})$ were grown overnight in Luria Bertani medium and then scaled up to $1 \mathrm{~L}$. Chloroamphenicol and 
carbenicillin were added to final concentrations of 34 and $100 \mu \mathrm{L}$, respectively. Protein expression was induced by the addition of $0.5 \mathrm{mM}$ isopropyl- $\beta$-D-thiogalactopyranoside (IPTG) at $\mathrm{OD}_{600}$ of $0.7-0.9$, and the cells incubated for an additional $3-4 \mathrm{~h}$ at $37^{\circ} \mathrm{C}$. Cells were harvested by centrifugation at $5000 \mathrm{~g}$ for $10 \mathrm{~min}$ at $4{ }^{\circ} \mathrm{C}$ and resuspended in lysis buffer consisting of $50 \mathrm{mM}$ Tris, $\mathrm{pH} 8.0,0.1 \%$ Triton X-100, and $1 \mathrm{mM}$ phenylmethylsulfonyl fluoride (PMSF). The cells were frozen at $-20{ }^{\circ} \mathrm{C}$ and lysed by thawing in lukewarm water.

Crude cell extract was obtained by ultracentrifugation at $102000 \mathrm{~g}$ for $1.5 \mathrm{~h}$. Purification was performed at $4{ }^{\circ} \mathrm{C}$. The sensor domain of $\mathrm{MmoS}$ was purified by nickel affinity chromatography on a chelating Sepharose column equilibrated with $50 \mathrm{mM}$ Tris, $\mathrm{pH} 8.0,0.5 \mathrm{M} \mathrm{NaCl}$, and $10 \%$ glycerol (buffer A). Approximately $100 \mathrm{~mL}$ of crude cell extract was loaded onto the column. The column was washed with $50 \mathrm{mM}$ Tris $\mathrm{pH} 8.0,0.5 \mathrm{M} \mathrm{NaCl}, 10 \%$ glycerol, and $15 \%$ of 0.5 $\mathrm{M}$ imidazole (buffer B). The sensor domain eluted at $50 \%$ buffer B ( $250 \mathrm{mM}$ imidazole) using a $600 \mathrm{~mL}$ gradient. Fractions containing the sensor domain were judged for purity by sodium dodecyl sulfate polyacrylamide gel electrophoresis (SDS-PAGE). Pure samples were pooled and concentrated using an Amicon $10 \mathrm{kD}$ centrifugal device (Millipore). Prior to crystallization, the protein was dialyzed overnight into $10 \mathrm{mM}$ Tris, $\mathrm{pH} 8.0,100 \mathrm{mM} \mathrm{NaCl}$. The (His) 6 tag was not removed.

\section{Crystallization and Data Collection}

Crystals of the sensor domain containing the FAD cofactor were grown by both hanging and sitting drop methods in a $20^{\circ} \mathrm{C}$ incubator. Bright yellow, rectangular crystals that were $1 \mathrm{~mm}$ in the longest dimension appeared within four days. Crystals grew at protein concentrations of $20-40 \mathrm{mg} / \mathrm{mL}$ with $0.3 \mathrm{M}$ ammonium phosphate and $8 \%$ glycerol as a precipitant. A cryoprotectant solution consisting of $15 \mu \mathrm{L} 0.3 \mathrm{M}$ ammonium phosphate and $35 \%$ glycerol plus $5 \mu \mathrm{l} 0.3 \mathrm{M}$ ammonium phosphate and $8 \%$ glycerol was added directly to the $2 \mu \mathrm{L}$ drop because the crystals were not stable upon exposure to air. The crystals were then frozen immediately in liquid nitrogen. Diffraction data were collected at $100 \mathrm{~K}$ using Mar225 and Mar300 CCD detectors at LS-CAT (sector 21) beamline ID-D and at GM/CA-CAT (sector 23) beamline ID-D, respectively, at the Advanced Photon Source (APS), Argonne, IL. All datasets were integrated and scaled with HKL2000 (23). There is one molecule in the asymmetric unit and the crystals belong to space group $P 42{ }_{1} 2$ with cell dimensions of $a=b=146.7 \AA$ and $c=$ $43.6 \AA$.

\section{Structure Determination}

The structure was solved by multiple isomorphous replacement with anomalous scattering (MIRAS) phasing using autoSHARP (24). Gold and tungsten derivatives were obtained by soaking crystals in $10 \mathrm{mM} \mathrm{KAu}(\mathrm{CN})_{2}$ and $10 \mathrm{mM} \mathrm{Na}_{2} \mathrm{WO}_{4}$, respectively, for $24 \mathrm{~h}$. An iodide derivative was obtained by soaking crystals in $0.3 \mathrm{M} \mathrm{KI}$ for $10 \mathrm{~s}$. Heavy atom solutions (1 $\mu \mathrm{L}$ ) were added directly to the crystallization drops. Soaking in heavy metals significantly decreased the resolution of the derivatized crystals (Table 1). Heavy atom sites (one Au, 11 $\mathrm{W}$, and three I) were located using SHELXD and phases were calculated using autoSHARP (24-26). An initial MIRAS experimental map was calculated to $2.5 \AA$ resolution and improved by solvent flattening using SOLOMON in autoSHARP with an estimated solvent content of $67.5 \%$.

\section{Model Building and Refinement}

Model building was initiated by placing the NifL N-terminal PAS domain coordinates (PDB accession code 2GJ3) (27) into the experimental map using MOLREP (28). This model facilitated the building of PAS-A. The rest of the structure was built manually in Coot (29). The FAD cofactor was obtained from the Coot library and modeled into the density. An initial polyalanine chain was modeled and subjected to iterative rounds of refinement, rebuilding, and 
sequence fitting using CNS (30) and Coot (29). The R-free remained stuck at $\sim 0.40$, however. After scaling the native data to $2.34 \AA$ resolution using the Diffraction Anisotropy Server (31), additional side chain density was visible and the sequence could be fitted correctly. This model was further refined with Refmac5 (32). The final model includes residues 99-325, one FAD molecule, 59 water molecules, one glycerol molecule, and one chloride ion (Table 1). A Ramachandran plot calculated with PROCHECK (33) indicates that $95.4 \%$ of the residues are in the most favored and additionally allowed regions. Figures were generated with PyMOL (34). Coordinate superpositions were performed using the LSQKAB program (35) or the SSM server (http://www.ebi.ac.uk/msd-srv/ssm) (36). Sequences were aligned using ClustalW in Jalview (37). Protein-protein interfaces were analyzed with PROTORP (http://www.bioinformatics.sussex.ac.uk/protorp).

\section{Gel Filtration Chromatography}

The molecular mass of the MmoS sensor domain was determined using a Superdex 200 gel filtration column (Amersham). The void volume was measured using a fresh solution of Blue Dextran $2000(1.0 \mathrm{mg} / \mathrm{mL})$ in buffer A. Molecular mass standards (Amersham) used to calibrate the column were: ferritin, $440 \mathrm{kDa}$; catalase, $232 \mathrm{kDa}$; aldolase, $158 \mathrm{kDa}$; albumin, $67 \mathrm{kDa}$; ovalbumin, $43 \mathrm{kDa}$, chymotrypsin, $25 \mathrm{kDa}$; ribonuclease A, $13.7 \mathrm{kDa}$. $K_{\mathrm{av}}$ values for each protein were calculated as described previously (10).

\section{Analytical Ultracentrifugation}

Sedimentation velocity experiments were carried out in the Keck facility at Northwestern University using a Beckman Coulter XL-A analytical ultracentrifuge. A $20 \mu \mathrm{M}$ sensor domain sample $(200 \mu \mathrm{L})$ was prepared in Buffer A and loaded into a 6 chamber charcoal centerpiece with Buffer A used as a reference solution. SV was performed at a rotor speed of 55,000 rpm at $12{ }^{\circ} \mathrm{C}$ for $595 \mathrm{~min}$. Samples were monitored at $280 \mathrm{~nm}$ and radial scans were collected every $120 \mathrm{~s}$. Data editing, hydrodynamic corrections for buffer density and viscosity and subsequent processing were performed using UltraScan 9.9 (38). A preliminary van Holde - Weischet analysis was used to obtain a model-independent and diffusion corrected range for the sedimentation coefficients for each sample (39). The value of the range was used to initialize a 2-dimensional spectrum analysis (2DSA) (40). Simultaneously, all systematic noise was removed from these data (41). All subsequent analyses were performed on modified datasets with all time invariant and radially invariant noise contributions subtracted. Further refinement of the data was achieved by performing a 2DSA Monte Carlo (2DSA-MC) analysis with 50 iterations (42). The 2DSA-MC data were used to initialize genetic algorithm analysis (GA) (43) which was performed with 50 Monte Carlo iterations.

\section{RESULTS AND DISCUSSION}

\section{Overall Structure}

The structure of the MmoS redox sensor was refined to 2.34 A resolution (Table 1). A (His) 6 tag and an enterokinase cleavage site were not visible in the electron density map nor were the first 14 and last four residues. There are no interactions between PAS-A and PAS-B. The two PAS domains (PAS-A and PAS-B) are linked by a long $\alpha$ helix ( $\alpha 4$, residues 203-216). Both domains exhibit the typical PAS domain $\alpha / \beta$ topology (1) (Figure 2A). Each domain contains an $\mathrm{N}$-terminal $\beta$ hairpin, $\beta 1$ (residues 100-104) and $\beta 2$ (residues 108-113) in PAS-A, and $\beta 6$ (residues 221-225) and $\beta 7$ (residues 231-234) in PAS-B. These two $\beta$-strands are followed by three $\alpha$-helices: $\alpha 1$ (residues 114-120), $\alpha 2$ (residues 124-127), and $\alpha 3$ (residues 142-152) in PAS-A and $\alpha 5$ (residues 236-242), $\alpha 6$ (residues 247-250), and $\alpha 7$ (residues 264-276) in PASB. There are short $3_{10}$ helices between helices $\alpha 2$ and $\alpha 3$ (residues 132-135) and between helices $\alpha 6$ and $\alpha 7$ (residues 254-256). The fold is completed by three additional $\beta$-strands: $\beta 3$ (residues 158-165), $\beta 4$ (residues 171-182), and $\beta 5$ (residues 188-197) in PAS-A and $\beta 8$ 
(residues 280-289), $\beta 9$ (residues 297-310), and $\beta 10$ (residues 316-324) in PAS-B. The two domains superpose with an rmsd of $2.05 \AA$ for $95 \mathrm{C} \alpha$ atoms. Some variations occur in the loop regions. The loops connecting $\beta 1$ and $\beta 2$ and $\beta 4$ and $\beta 5$ in PAS-A adopt different conformations than their counterparts in PAS-B. The most significant difference is that the loop connecting $\beta 3$ and $\beta 4$ in PAS-A is shorter than that connecting $\beta 8$ and $\beta 9$ in PAS-B (Figure 2A).

The only other structure of a tandem PAS domain is that of a fragment of the Drosophila circadian clock protein PERIOD (dPER) (44). dPER does not contain a cofactor. In the dPER structure, the two PAS domains are arranged in a completely different fashion from MmoS. The two dPER PAS domains are linked by a loop region rather than an $\alpha$ helix and interact with one another (Figure 2B). In particular, $\sim 700 \AA^{2}$ of surface area is buried by an interaction by the three C-terminal $\beta$-strands of PAS-A and the N-terminal helices of PAS-B (44). The analogous $\beta$-strands in the MmoS sensor PAS-A interact with the $\beta$-sheet of PAS-B from a symmetry related molecule. Also relevant is a structure of the sensor domain of DctB, a tandem PDC domain that senses $\mathrm{C}_{4}$-dicarboxylates (45). PDC domains are similar to PAS domains, but are distinguished by an $\mathrm{N}$-terminal $\alpha$ helix and different secondary structure connecting the $\beta$ strands (46). In the DctB structure, the two PDC domains are linked by an $\alpha$-helix, similar to the $\mathrm{MmoS}$ sensor, but an extended $\mathrm{N}$-terminal $\alpha$-helix, not present in $\mathrm{MmoS}$, holds the two domains in close proximity (45).

\section{FAD Binding Site}

A single FAD molecule is present in PAS-A consistent with the previously reported stoichiometry of one FAD per MmoS (10). Although PAS-A and PAS-B are 24\% identical and 30\% similar (comparing residues 85-201 and 209-329), residues involved in binding FAD in PAS-A (Figure 3) are not well conserved in PAS-B (Figure 4B). The isoalloxazine ring is located within a deep cavity with the ribityl chain and adenosine extending into a groove on the protein surface. PAS-B lacks a similar surface groove, and has a smaller internal cavity that does not open to the surface. An extended network of hydrogen bonds, van der Waals, and electrostatic interactions involving primarily residues from $\alpha 2, \alpha 3$, and $\beta 3$ hold the FAD in place. In addition, several residues from $\alpha 7$ of PAS-B in a symmetry related molecule are involved (Figure 3). The $\mathrm{O} 4$ and N3 atoms of the isoalloxazine ring interact with the side chain of Asn 164, and a water molecule hydrogen bonded to the $\mathrm{O} 2$ atom interacts with the side chain of Asn 136. The side chain of His 132 is $3.2 \AA$ from the N5 atom, and a water molecule hydrogen bonded to the N5 atom links it to the side chain of Ser 193. In addition, the sulfur atom of Met 148 is within $6 \AA$ of the isoalloxazine ring and could modulate the redox potential (47). The ribityl chain is in the gauche conformation and is stabilized by interactions with water molecules, Arg 133, Lys 142, and the side chain of Glu 272 in the symmetry related PAS-B domain. The ribose ring interacts with Glu 265 in the symmetry related PAS-B via an intervening water molecule. Finally, the Trp 149 side chain stacks on the adenine ring.

\section{Comparison to Other PAS domains}

Secondary structure comparisons performed with the SSM server (http://www.ebi.ac.uk/msd-srv/ssm) (36) indicate that the MmoS sensor PAS-A domain is most similar to the $\mathrm{N}$-terminal, FAD-containing PAS domain of Azotobacter vinelandii NifL (27) with an rmsd of $1.13 \AA$ for $101 \mathrm{C} \alpha$ coordinates. Other close matches include the FMNcontaining plant photoreceptor domains (Figure 4A). For example, PAS-A superposes with the Chlamydomonas reinhardtii phototropin LOV1 domain (48) with an rmsd of $1.60 \AA$ for $100 \mathrm{C} \alpha$ coordinates and the phy3 LOV2 domain (49) with an rmsd of $1.69 \AA$ for $98 \mathrm{C} \alpha$ atoms. The overall sequence identities between MmoS PAS-A and these domains is $20-35 \%$ (Figure 4B). Similar results are obtained for PAS-B, with rmsd values of 1.63 and $1.68 \AA$ for phy 3 LOV2 and C. reinhardtii LOV1, respectively. PAS-B is less similar to NifL, with an rmsd of $2.00 \AA$ for $96 \mathrm{C} \alpha$ atoms (Figure 4A). The MmoS sensor domain is sequentially most similar 
to NifL and Aer, which also binds FAD (50). Residues that interact with FAD in MmoS are well conserved in NifL (27). Notable exceptions are His 132 and Ser 193, of which the NifL counterparts are glutamic acid and glycine, respectively. In NifL, Ser 39 interacts with the FAD via water molecules. The corresponding PAS-A residue, Ser 101, is in a similar position, but interacting water molecules are not observed, perhaps due to the lower resolution of the structure. The MmoS PAS domains are also fairly similar to the heme-containing sensor domains E. coli DosH (51) and Bradyrhizobium japonicum FixLH (Figure 4B) (52). A major difference in these domains is the position of the helix corresponding to PAS-A $\alpha 3$, which is altered to accommodate a heme rather than an FAD cofactor (Figure 4A).

\section{Oligomerization State}

According to previous gel filtration studies, intact $\mathrm{MmoS} \mathrm{Tm}^{-}$forms a tetramer (10), similar to $\operatorname{NifL}(53,54)$. Gel filtration indicates that the sensor domain is dimeric (Figure S1). Analytical ultracentrifugation reveals the presence of two main species in equilibrium, a monomer of approximate molecular weight $22.8 \mathrm{kDa}$ representing $33.7 \%$ of the total protein concentration and a dimer of approximate molecular weight $54.6 \mathrm{kDa}$ accounting for $61.3 \%$ of the total protein concentration. The remaining $5 \%$ is present as higher oligomers (Figure S2 and Table S1). The crystallographic asymmetric unit contains a monomer, however. In the crystal lattice, there are two types of interaction surfaces between monomers (Figure 5). First, the FAD binding site in PAS-A from one monomer interacts with PAS-B helix $\alpha 7$ from a symmetry related monomer (interface 1). Besides the aforementioned interactions between Glu 265 and Glu 272 and the FAD, hydrogen bonds link Asp 131 with Gln 275, Gln 106 with Gln 264, and Arg 108 with Ala 228 and Gln 271. The interface accessible surface area buried per monomer is $484 \AA^{2}$, which is low for a stable or even transient dimer (55). In the second intermonomer surface, strands $\beta 3, \beta 4$, and $\beta 5$ from PAS-A, which lie on the opposite side of the molecule from the FAD, interact primarily with the connecting helix $\alpha 4$ and the $\mathrm{N}$-terminus of PAS-B from a symmetry related monomer (interface 2). This interface constitutes $883 \AA^{2}$ buried surface area and involves a hydrophobic face of $\alpha 4$, including residues Ala 204, Leu 211, Ala 214, Met 215, and Ala 217. In addition, unidentified electron density that was partially modeled as a glycerol molecule is present at this interface.

Two types of dimers have been observed in other PAS domain structures. The N-terminal PAS domain of NifL forms a homodimer in which an N-terminal amphipathic $\alpha$ helix from one monomer packs against the hydrophobic surface of the $\beta$ sheet in the second monomer (27). The cofactors lie on the opposite sides on the dimer, remote from the interface. This arrangement, also observed in the heme sensors DosH (51) and FixLH (56) and several other structures (27), has been proposed to represent a general phenomenon for flavin and heme binding sensors (27). Unlike this dimer, the FAD lies at interface 1 in the MmoS sensor domain structure and the $\mathrm{N}$-terminus is disordered. Interface 2 is more similar to the NifL dimer in that the PAS-A $\beta$ sheet opposite the FAD is involved and interacts with a helix. The helix is the connecting helix to PAS-B in another monomer rather than the N-terminal helix of another PAS-A as in NifL, however. Neither interface in the MmoS sensor domain structure is as extensive as that in NifL, which buries $2066 \AA^{2}$ solvent accessible surface area (27).

The second type of dimer observed in PAS domain structures is that in the dPER structure (44). In this structure, a noncrystallographic dimer of tandem PAS domains is present. In addition to the dPER intramolecular interactions mentioned above (Figure 2B), the C-terminal helix in PAS-B from one monomer interacts with the $\beta$ sheet of PAS-A from the second monomer. The nature of the interaction is somewhat similar to interface 2, but the helix derives from the $\mathrm{C}$-terminus rather than the linker region. Moreover, this $\mathrm{C}$-terminal region is part of a conserved C-domain specific to circadian clock proteins $(44,57)$. The dPER dimer is also more extensive, with $1300 \AA^{2}$ buried surface area. Given the nature and limited extent of the 
MmoS sensor domain crystallographic interfaces and the differences from the NifL and dPER dimers, it seems unlikely that a physiological dimer is present. Since analytical ultracentrifugation data suggest that the $\mathrm{MmoS}$ sensor domain exists in a monomer-dimer equilibrium, it may be that crystallization favors the monomeric form. Formation of tetramers in intact $\mathrm{MmoS}$ (10) may require the $\mathrm{C}$-terminal domains. In addition, the $\mathrm{N}$-terminal transmembrane helix and interaction with the membrane could affect the oligomerization state in vivo.

\section{Functional Implications}

The nature of the redox signal detected by the MmoS sensor domain is not known. Possibilities include copper, the copper chelator methanobactin (58), quinones, and reactive oxygen species (10). In the simplest model, oxidation of $\mathrm{FADH}_{2}$ to $\mathrm{FAD}$ could be coupled directly to the reduction of aqueous $\mathrm{Cu}(\mathrm{II})$ to $\mathrm{Cu}(\mathrm{I})\left(E_{o}=150 \mathrm{mV}\right)$. However, copper in the cytoplasm is most likely $\mathrm{Cu}(\mathrm{I})$. Alternatively, quinones or methanobactin could sense copper and modulate the redox state of the FAD cofactor. Finally, reactive oxygen species generated by copper could play a role in signaling MmoS. Since MmoS contains an N-terminal transmembrane region, PAS-A likely resides near the membrane. Interestingly, residue Trp 158 is within $10 \AA$ of Trp 149 which is within $6 \AA$ of the FAD isoalloxazine ring. It is plausible that these two tryptophans mediate electron transfer from diffusible membrane electron carriers such as quinones. Other important players in the redox chemistry at the FAD cofactor may be water molecules, as suggested for NifL (27), and His 132. The NifL residue equivalent to His 132 is a glutamic acid and is proposed to interact with a hydroperoxy intermediate at the $\mathrm{C} 4 \mathrm{a}$ atom of FAD (27). In the MmoS sensor domain, His 132 could play a role in protonation and deprotonation of intermediate species or water molecules. In LOV2, the residue corresponding to His 132 is a cysteine that forms a light-induced, covalent flavin-cysteinyl adduct $(49,59)$. Interestingly, histidine residues are covalently linked to the FAD cofactor in some enzymes (60).

Protonation of the N1 and N5 of FAD combined with any other modifications that occur upon reduction will at the very least lead to rearrangement of the hydrogen bonding pattern at the FAD site. It may be that reduction leads to major conformational changes as well. In support of this notion, all efforts to reduce crystals of the MmoS sensor domain significantly reduced diffraction quality, consistent with a large rearrangement. As suggested for NifL (27), changes accompanying reduction could be propagated to other regions of MmoS involved in proteinprotein interactions. In our previously proposed model (10), oxidized MmoS interacts with MmoR, inhibiting transcription of the sMMO genes. This interaction could either involve PASA and/or PAS-B directly or the other domains. For NifL, the linker region between the PAS domains and the $\mathrm{C}$-terminus mediates an interaction with the transcription factor NifA when the FAD is oxidized $(61,62)$. The PAS domains are not required for interaction with NifA. When the MmoS FAD is reduced to $\mathrm{FADH}_{2}$, the resultant conformational changes in the PAS domains may prevent interaction with MmoR, possibly through a change in oligomerization state. These changes might also convey a signal to the other domains, perhaps promoting autophosphorylation and interaction with MmoQ. Protein-protein interaction studies to test these ideas are underway.

\section{Supplementary Material}

Refer to Web version on PubMed Central for supplementary material.

\section{ACKNOWLEDGEMENTS}

Northwestern University is a member institution of the Life Sciences Collaborative Access Team (LS-CAT) at sector 21 of the Advanced Photon Source, Argonne, IL. GM/CA CAT has been funded in whole or in part with Federal funds from the National Cancer Institute (Y1-CO-1020) and the National Institute of General Medical Science (Y1- 
GM-1104). Use of the Advanced Photon Source was supported by the U.S. Department of Energy, Basic Energy Sciences, Office of Science, under contract No. DE-AC02-06CH1135. Analytical ultracentrifugation experiments were conducted using a Beckman Optima XLA centrifuge in the Northwestern University Keck Biophysics Facility.

This work was supported by NIH grant GM70473. U.E.U. was supported in part by NIH Training Grant GM8061.

\section{Abbreviations}

PAS, PER-ARNT-SIM

MMO, methane monooxygenase

sMMO, soluble methane monooxygenase

pMMO, particulate methane monooxygenase

FAD, flavin adenine dinucleotide

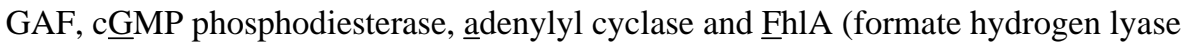
transcriptional activator) domain

HisKA, histidine kinase A phosphoacceptor domain

HATPase, histidine kinase ATPase domain

REC, phosphoreceiver domain

HPT, histidine phosphotransfer domain

IPTG, isopropyl- $\beta$-D-thiogalactopyranoside

PMSF, phenylmethylsulfonyl fluoride

MIRAS, multiple isomorphous replacement with anomalous scattering

r.m.s.d, root mean square deviation

\section{REFERENCES}

(1). Hefti M, Françoijs K-J, de Vries LC, Dixon R, Vervoort J. The PAS fold: a redefinition of the PAS domain based upon structural prediction. Eur. J. Biochem 2004;271:1198-1208. [PubMed: 15009198]

(2). Taylor BL, Zhulin IB. PAS domains: internal sensors of oxygen, redox potential, and light. Microbiol. Mol. Biol. Rev 1999;63:479-506. [PubMed: 10357859]

(3). Lindebro MC, Poellinger L, Whitelaw ML. Protein-protein interaction via PAS domains: role of the PAS domain in positive and negative regulation of the bHLH/PAS dioxin receptor-Arnt transcription factor complex. EMBO J 1995;14:3528-3539. [PubMed: 7628454]

(4). Ponting CP, Aravind L. PAS: a multifunctional domain family comes to light. Current Biol 1997;7:R674-R677.

(5). Stock AM, Robinson VL, Goudreau PN. Two-component signal transduction. Ann. Rev. Biochem 2000;69:183-215. [PubMed: 10966457]

(6). West AH, Stock AM. Histidine kinases and response regulator proteins in two-component signaling systems. Trends Biochem. Sci 2001;26:369-376. [PubMed: 11406410]

(7). Mizuno T. His-Asp phosphotransfer signal transduction. J. Biochem 1998;123:555-563. [PubMed: 9538242]

(8). Csáki R, Bodrossy L, Klem J, Murrell JC, Kovács KL. Genes involved in the copper-dependent regulation of soluble methane monooxygenase of Methylococcus capsulatus (Bath): cloning, sequencing and mutational analysis. Microbiol 2003;149:1785-1795.

(9). Hakemian AS, Rosenzweig AC. The biochemistry of methane oxidation. Ann. Rev. Biochem 2007;76:223-241. [PubMed: 17328677]

(10). Ukaegbu UE, Henery S, Rosenzweig AC. Biochemical characterization of MmoS, a sensor protein involved in copper-dependent regulation of soluble methane monooxygenase. Biochemistry 2006;45:10191-10198. [PubMed: 16922494]

(11). Hanson RS, Hanson TE. Methanotrophic bacteria. Microbiol. Rev 1996;60:439-471. [PubMed: 8801441]

(12). Dedysh SN, Knief C, Dunfield PF. Methylocella species are facultatively methanotrophic. J. Bacteriol 2005;187:4665-4670. [PubMed: 15968078] 
(13). Theisen AR, Ali MH, Radajewski S, Dumont MG, Dunfield PF, McDonald IR, Dedysh SN, Miguez $\mathrm{CB}$, Murrell JC. Regulation of methane oxidation in the facultative methanotroph Methylocella silvestris BL2. Mol. Microbiol 2005;58:682-692. [PubMed: 16238619]

(14). Balasubramanian R, Rosenzweig AC. Structural and mechanistic insights into methane oxidation by particulate methane monooxygenase. Acc. Chem. Res 2007;40:573-580. [PubMed: 17444606]

(15). Merkx M, Kopp DA, Sazinsky MH, Blazyk JL, Müller J, Lippard SJ. Dioxygen activation and methane hydroxylation by soluble methane monooxygenase: a tale of two irons and three proteins. Angew. Chem. Int. Ed 2001;40:2782-2807.

(16). Murray LJ, Lippard SJ. Substrate trafficking and dioxygen activation in bacterial multicomponent monooxygenases. Acc. Chem. Res 2007;40:466-474. [PubMed: 17518435]

(17). Prior SD, Dalton H. The effect of copper ions on membrane content and methane monooxygenase activity in methanol-grown cells of Methylococcus capsulatus (Bath). J. Gen. Microbiol 1985;131:155-163.

(18). Stanley SH, Prior SD, Leak DJ, Dalton H. Copper stress underlies the fundamental change in intracellular location of methane monooxygenase in methane oxidizing organisms: studies in batch and continuous cultures. Biotechnol. Lett 1983;5:487-492.

(19). Stafford GP, Scanlan J, McDonald IR, Murrell JC. rpoN, $m m o R$ and $m m o G$, genes involved in regulating the expression of soluble methane monooxygenase from Methylosinus trichosporium OB3b. Microbiol 2003;149:1771-1784.

(20). Aravind L, Ponting CP. The GAF domain: an evolutionary link between diverse phototransducing proteins. Trends Biochem. Sci 1997;22:458-459. [PubMed: 9433123]

(21). Martinez-Argudo I, Little R, Shearer N, Johnson P, Dixon R. The NifL-NifA System: a multidomain transcriptional regulatory complex that integrates environmental signals. J. Bacteriol 2004;186:601-610. [PubMed: 14729684]

(22). Taylor BL, Rebbapragada A, Johnson MS. The FAD-PAS domain as a sensor for behavioral responses in Escherichia coli. Antioxid. Redox Signal 2001;3:867-879. [PubMed: 11761333]

(23). Otwinowski Z, Minor W. Processing of X-ray diffraction data collected in oscillation mode. Methods Enzymol 1997;276:307-326.

(24). Vonrhein, C.; Blanc, E.; Roversi, P.; Bricogne, G. Automated structure solution with autoSHARP. In: Doublié, S., editor. Methods in Molecular Biology. Humana Press, Inc.; Totowa, NJ: 2007. p. 215-230.

(25). de la Fortelle E, Bricogne G. Maximum-likelihood heavy-atom parameter refinement for multiple isomorphous replacement and multiwavelength anomalous diffraction methods. Meth. Enz 1997;276:472-494.

(26). Schneider TR, Sheldrick GM. Substructure solution with SHELXD. Acta Cryst 2002;D58:17721779.

(27). Key J, Hefti M, Purcell EB, Moffat K. Structure of the redox sensor domain of Azotobacter vinelandii NifL at atomic resolution: signaling, dimerization, and mechanism. Biochemistry 2007;46:3614-3623. [PubMed: 17319691]

(28). Vagin, A. t.; Teplyakov, A. MOLREP: an automated program for molecular replacement. J. Appl. Crystallogr 1997;30:1022-1025.

(29). Emsley P, Cowtan K. Coot: model-building tools for molecular graphics. Acta Cryst 2004;D60:2126-2132.

(30). Brünger AT, Adams PD, Clore GM, DeLano WL, Gros P, Grosse-Kunstleve RW, Jiang J-S, Kuszewski J, Nilges M, Pannu NS, Read RJ, Rice LM, Simonson T, Warren GL. Crystallography \& NMR system: a new software suite for macromolecular crystallography. Acta Cryst 1998;D54:905-921.

(31). Strong M, Sawaya MR, Wang S, Phillips M, Cascio D, Eisenberg D. Toward the structural genomics of complexes: crystal structure of a PE/PPE protein complex from Mycobacterium tuberculosis. PNAS 2006;103:8060-8065. [PubMed: 16690741]

(32). Murshudov GN, Vagin AA, Dodson EJ. Refinement of macromolecular structures by the maximumlikelihood method. Acta Cryst 1997;D53:240-255.

(33). Laskowski RA. PROCHECK: a program to check the stereochemical quality of protein structures. J. Appl. Cryst 1993;26:283-291. 
(34). Delano, WL. The PyMOL molecular graphics system. DeLano Scientific; San Carlos, CA: 2002.

(35). Collaborative Computational Project Number 4. The CCP4 suite programs for protein crystallography. Acta Cryst 1994;D50:760-763.

(36). Krissinel E, Henrick K. Secondary-structure matching (SSM), a new tool for fast protein structure alignment in three dimensions. Acta Cryst 2004;D60:2256-2268.

(37). Clamp M, Cuff J, Searle SM, Barton GJ. The Jalview Java alignment editor. Bioinformatics 2004;20:426-427. [PubMed: 14960472]

(38). Demeler, B. Ultrascan 9.9: a comprehensive data analysis software package for analytical ultracentrifugation experiments. The University of Texas Health Science Center at San Antonio, Department of Biochemistry; 2008. http://www.ultrascan.uthscsa.edu

(39). Demeler B, van Holde KE. Sedimentation velocity analysis of heterogeneous systems. Anal. Biochem 2004;335:279-288. [PubMed: 15556567]

(40). Brookes, E.; Boppana, RV.; Demeler, B. Supercomputing '06 ACM 0-7695-2700-0/06. 2006. Computing large sparse multivariate optimization problems with an application in biophysics.

(41). Schuck P, Demeler B. Direct sedimentation boundary analysis of interference optical data in analytical ultracentrifugation. Biophys. J 1999;76:2288-2296. [PubMed: 10096923]

(42). Demeler B, Brookes E. Monte Carlo analysis of sedimentation experiments. Colloid Polym. Sci 2008;286:129-137.

(43). Brookes, E.; Demeler, B. Algorithm optimization for obtaining accurate molecular weight distributions from sedimentation velocity experiments. In: Wandrey, C.; Cölfen, H., editors. Analytical Ultracentrifugation VIII, Progr. Colloid Polym. Sci. Springer; 2006. p. 78-82.

(44). Yildiz O, Doi M, Yujnovsky I, Cardone L, Berndt A, Hennig S, Schulze S, Urbanke C, SassoneCorsi P, Wolf E. Crystal structure and interactions of the PAS repeat region of the Drosophila clock protein PERIOD. Mol. Cell 2005;17:69-82. [PubMed: 15629718]

(45). Zhou Y-F, Nan B, Nan J, Ma Q, Panjikar S, Liang Y-H, Wang Y, Su X-D. C4-dicarboxylates sensing mechanism revealed by the crystal structures of DctB sensor domain. J. Mol. Biol 2008;383:49_ 61. [PubMed: 18725229]

(46). Cheung J, Bingman CA, Reyngold M, Hendrickson WA, Waldburger CD. Crystal structure of a functional dimer of the PhoQ sensor domain. J. Biol. Chem 2008;283:13762-13770. [PubMed: 18348979]

(47). Druhan LJ, Swenson RP. Role of methionine 56 in the control of the oxidation-reduction potentials of the Clostridium beijerinckii flavodoxin: effects of substitutions by aliphatic amino acids and evidence for a role of sulfur-flavin interactions. Biochemistry 1998;37:9668-9678. [PubMed: 9657679]

(48). Fedorov R, Schlichting I, Hartmann E, Domratcheva T, Fuhrmann M, Hegemann P. Crystal structures and molecular mechanism of a light-induced signaling switch: the Phot-LOV1 domain from Chlamydomonas reinhardtii. Biophys. J 2003;84:2474-2482. [PubMed: 12668455]

(49). Crosson S, Moffat K. Photoexcited structure of a plant photoreceptor domain reveals a light-driven molecular switch. The Plant Cell 2002;14:1067-1075. [PubMed: 12034897]

(50). Bibikov SI, Barnes LA, Gitin Y, Parkinson JS. Domain organization and flavin adenine dinucleotide-binding determinants in the aerotaxis signal transducer Aer of Escherichia coli. Proc. Natl. Acad. Sci. USA 2000;97:5830-5835. [PubMed: 10811894]

(51). Park H, Suquet C, Satterlee JD, Kang C. Insights into signal transduction involving PAS domain oxygen-sensing heme proteins from the X-ray crystal structure of Escherichia coli Dos heme domain (Ec DosH). Biochemistry 2004;43:2738-2746. [PubMed: 15005609]

(52). Key J, Moffat K. Crystal structures of deoxy and CO-bound $b j$ FixLH reveal details of ligand recognition and signaling. Biochemistry 2005;44:4627-4635. [PubMed: 15779889]

(53). Hill S, Austin S, Eydmann T, Jones T, Dixon R. Azotobacter vinelandii NIFL is a flavoprotein that modulates transcriptional activation of nitrogen-fixation genes via a redox-sensitive switch. Proc. Natl. Acad. Sci. USA 1996;93:2143-2148. [PubMed: 8700899]

(54). Söderbäck E, Reyes-Ramirez F, Eydmann T, Austin S, Hill S, Dixon R. The redox- and fixed nitrogen-responsive regulatory protein NIFL from Azotobacter vinelandii comprises discrete flavin and nucleotide-binding domains. Mol. Microbiol 1998;28:179-192. [PubMed: 9593306] 
(55). Jones S, Thornton JM. Principles of protein-protein interactions. Proc. Natl. Acad. Sci. USA 1996;93:13-20. [PubMed: 8552589]

(56). Miyatake H, Mukai M, Park S-Y, Adachi S-I, Tamura K, Nakamura H, Nakamura K, Tsuchiya T, Iizuka T, Shiro I. Sensory mechanism of oxygen sensor FixL from Rhizobium meliloti: crystallographic, mutagenesis and resonance raman spectroscopic studies. J. Mol. Biol 2000;301:415-431. [PubMed: 10926518]

(57). Huang ZJ, Curtin KD, Roshbash M. PER protein interactions and temperature compensation of a circadian clock in Drosophila. Science 1995;267:1169-1172. [PubMed: 7855598]

(58). Balasubramanian R, Rosenzweig AC. Copper methanobactin: a molecule whose time has come. Curr. Op. Chem. Biol 2008;12:245-249.

(59). Crosson S, Rajagopal S, Moffat K. The LOV domain family: photoresponsive signaling modules coupled to diverse output domains. Biochemistry 2003;42:2-10. [PubMed: 12515534]

(60). Edmondson DE, Newton-Vinson P. The covalent FAD of monoamine oxidase: structural and functional role and mechanism of the flavinylation reaction. Antioxid. Redox Signal 2001;3:789806. [PubMed: 11761328]

(61). Money T, Jones T, Dixon R, Austin S. Isolation and properties of the complex between the enhancer binding protein NIFA and the sensor NIFL. J. Bacteriol 1999;181:4461-4468. [PubMed: 10419940]

(62). Xie Z, Dou Y, Ping S, Chen M, Wang G, Elmerich C, Lin M. Interaction between NifL and NifA in the nitrogen-fixing Pseudomonas stutzeri A1501. Microbiology 2006;152:3535-3542. [PubMed: 17159205] 


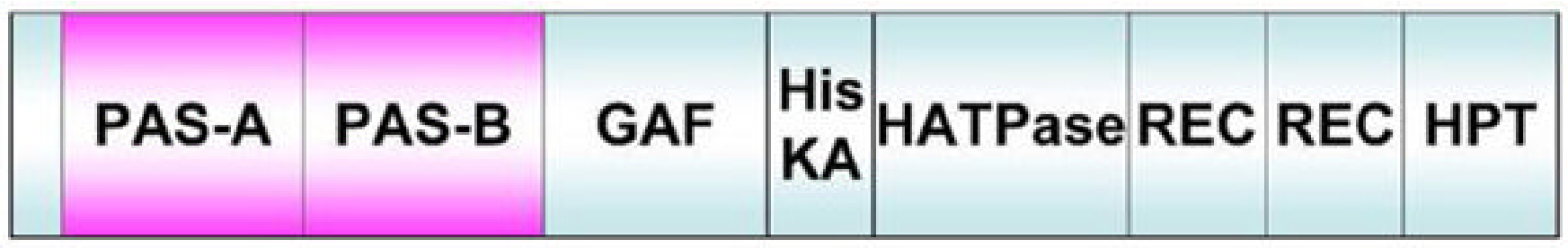

Figure 1.

Predicted domain organization of MmoS. The two PAS-PAC domains (labeled PAS-A and PAS-B) are followed by a GAF domain. The HATPase domain likely catalyzes phosphorylation of a histidine in the HisKA domain. The receiver domains (REC) and Hiscontaining phosphotransfer domain may function in phosphate transfer to the partner regulator protein MmoQ. 


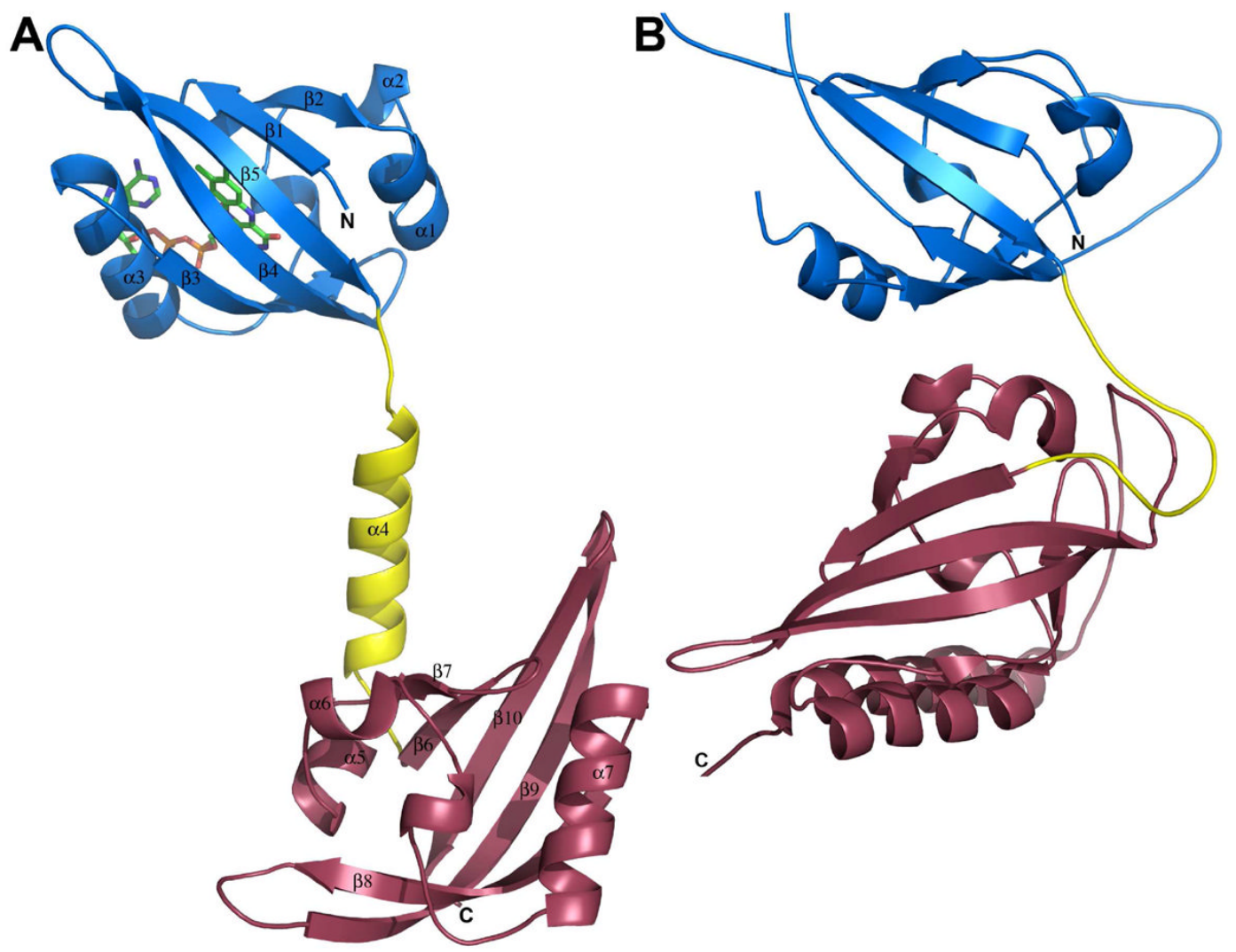

Figure 2.

Arrangement of the tandem PAS domains in (A) MmoS and (B) dPER (PDB accession code 1WA9). PAS-A is shown in blue, the linker region is shown in yellow, and PAS-B is shown in raspberry. The secondary structure elements are labeled for MmoS and the FAD cofactor is shown as sticks. 

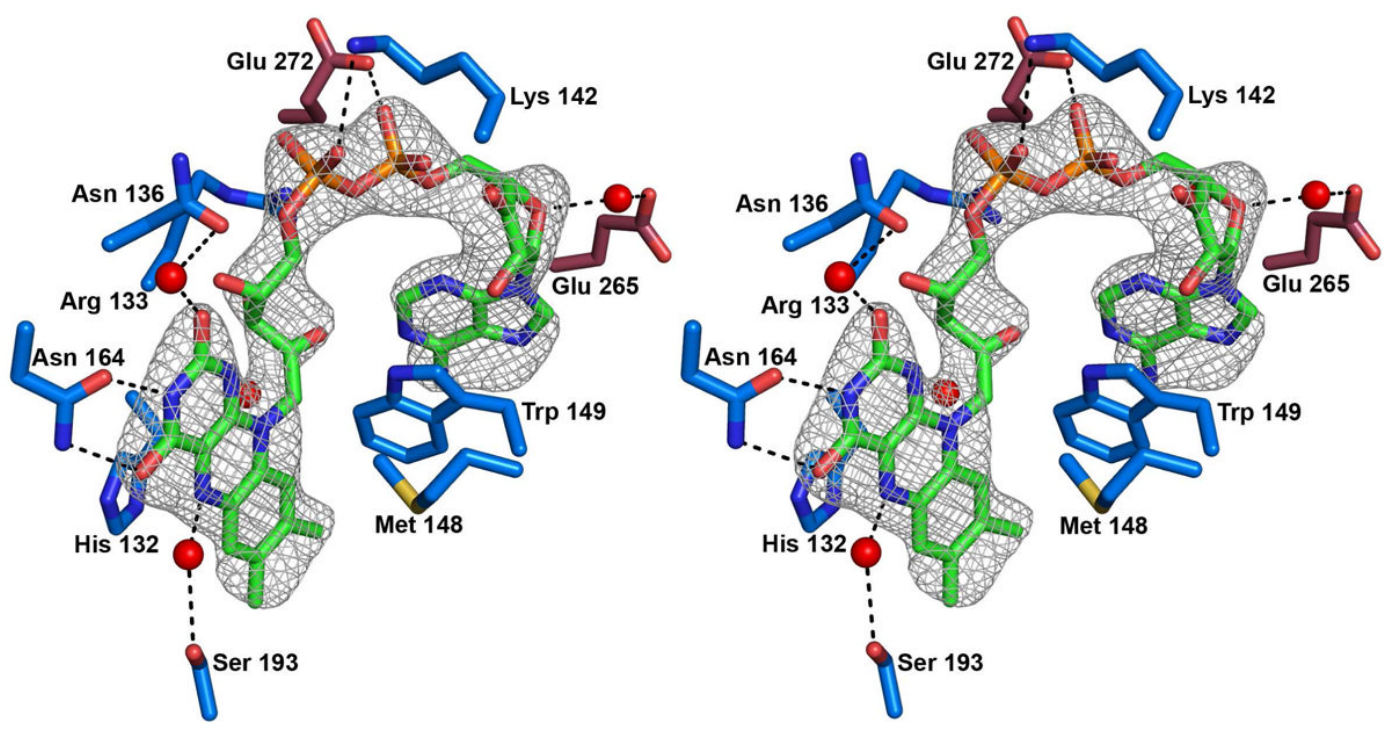

Figure 3.

Stereo view of the FAD cofactor in PAS-A and interacting residues. Residues from PAS-A have blue carbon atoms, residues from PAS-B have raspberry carbon atoms, and the FAD has green carbon atoms. Nitrogen atoms are blue, oxygen atoms are red, sulfur atoms are yellow, and phosphorus atoms are orange. Water molecules are shown as red spheres. An omit electron density map of the FAD contoured at $3 \sigma$ is superimposed. 


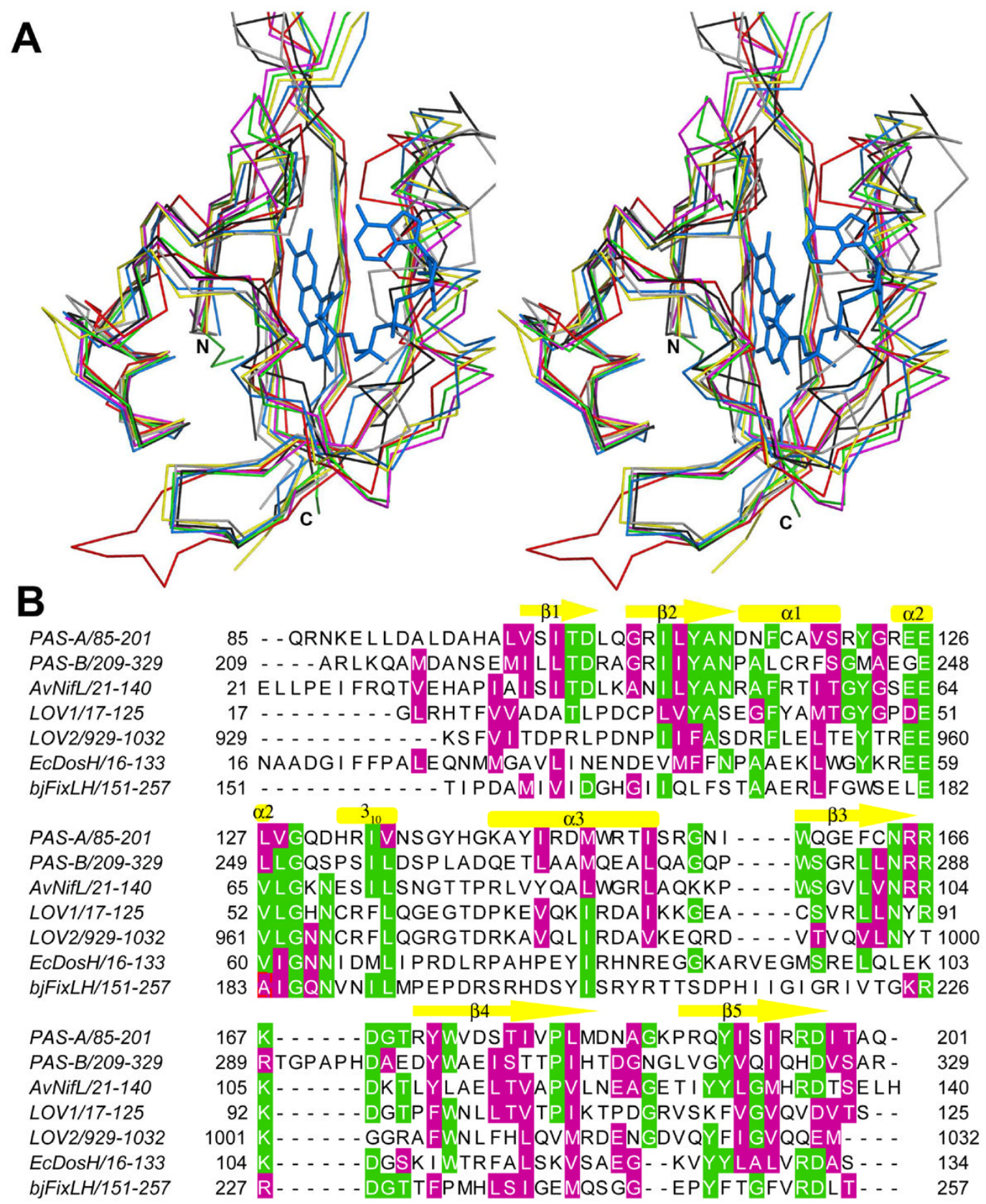

Figure 4.

Comparison of MmoS PAS domains to other PAS domains. (A) Superposition of PAS-A (blue), PAS-B (red), A. vinelandii NifL (yellow, PDB accession code 2GJ3), C. reinhardtii LOV1 (green, 1N9O), phy3 LOV2 (magenta, 1JNU), E. coli27DosH (black, 1V9Z), and $B$. japonicum FixLH (gray, 1XJ6). The FAD cofactor in PAS-A is shown as blue sticks. The heme cofactors in DosH and FixLH are not shown. (B) Structure based sequence alignment of structures shown in (A). Identical residues are highlighted green and similar residues are highlighted magenta. The secondary structure elements for MmoS PAS-A are indicated. 


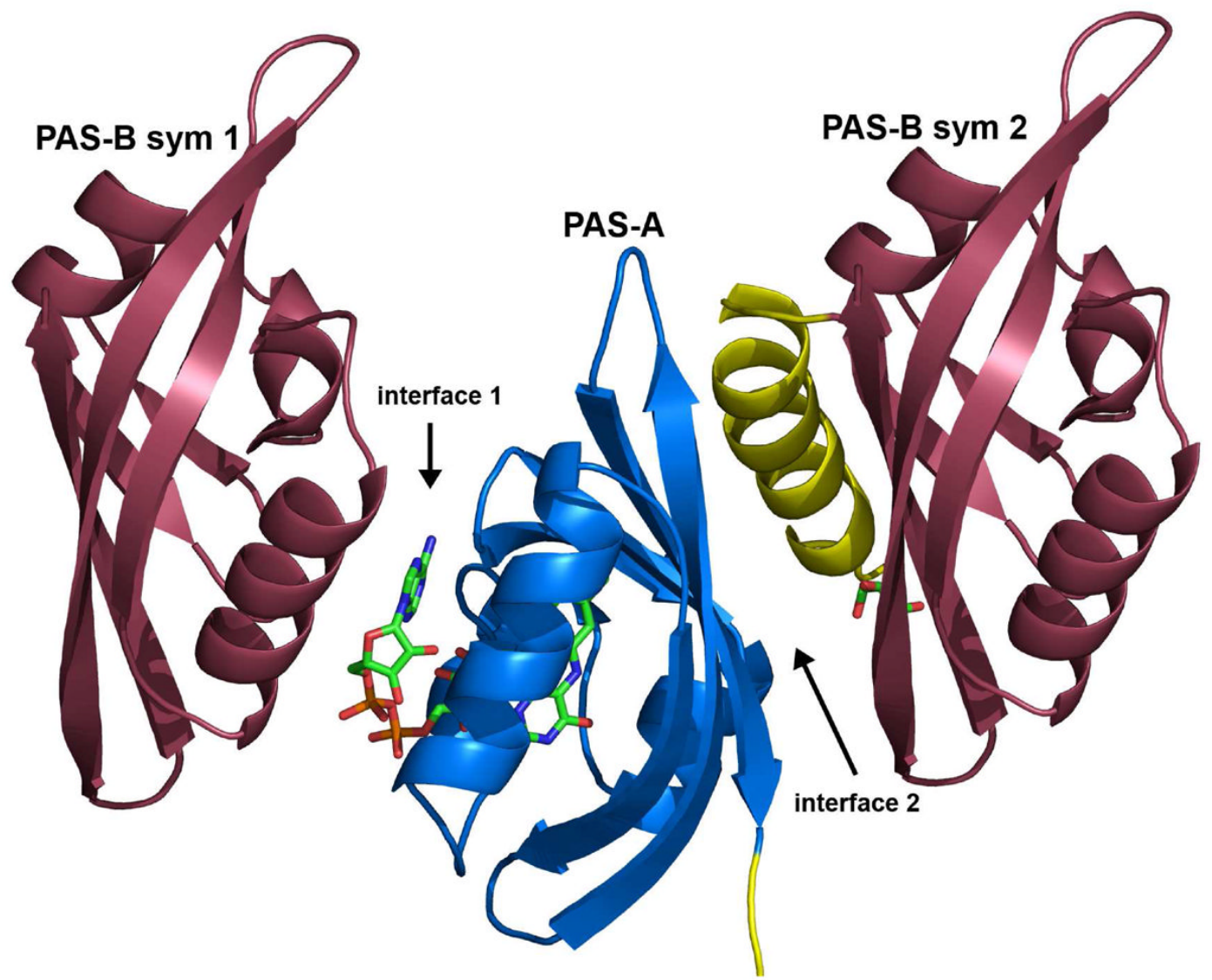

Figure 5.

Crystal packing interactions between symmetry related monomers. The PAS-A domain in the middle interacts with the PAS-B domains from two different symmetry related molecules via interface 1 and interface 2 . 


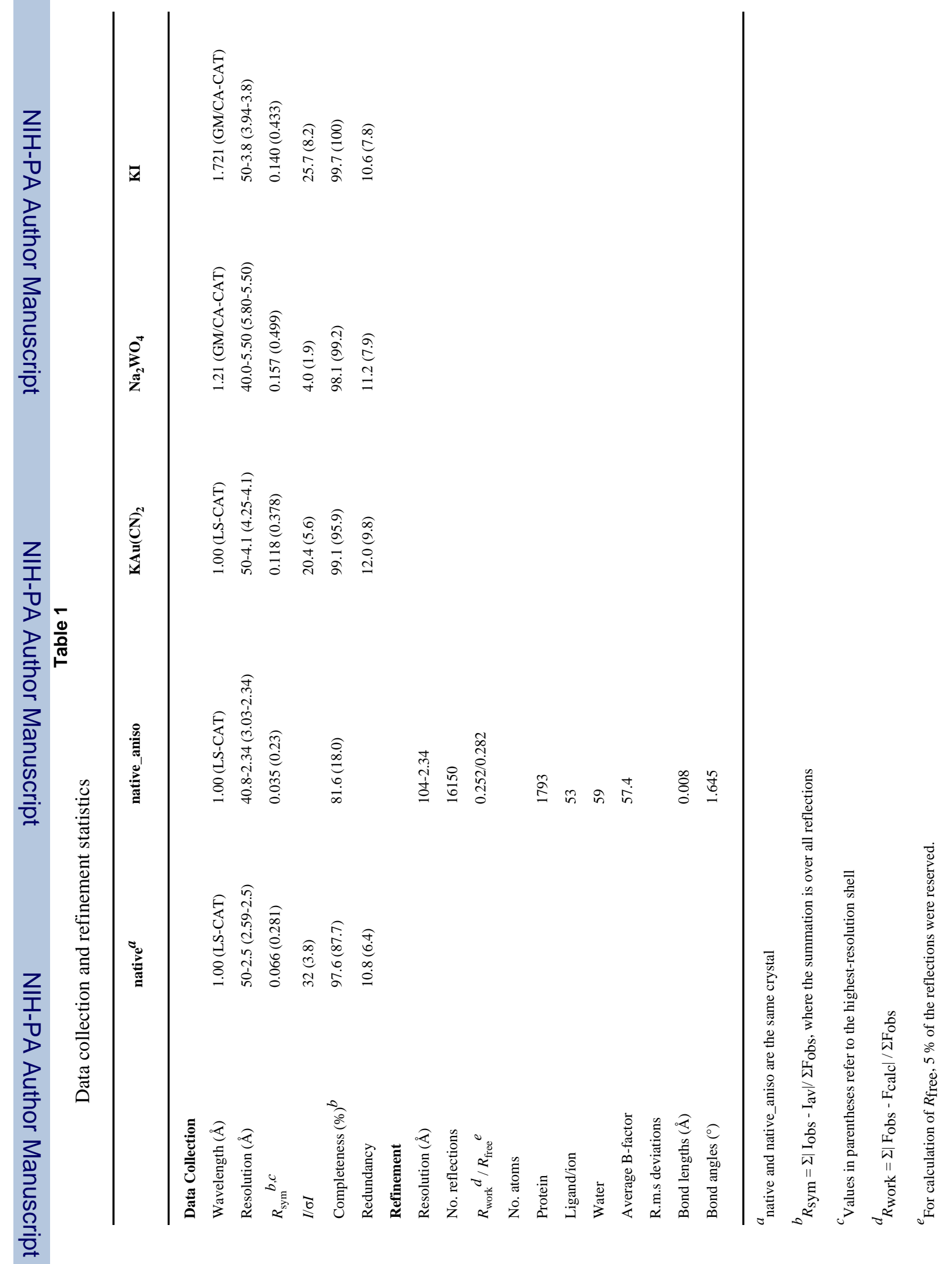

